TAMKANG JOURNAL OF MATHEMATICS

Volume 41, Number 4, 313-316, Winter 2010

\title{
THE CONTINUED FRACTION EXPANSION OF EULER'S CONSTANT
}

\section{STEFAN CZEKALSKI}

Abstract. The paper presents the continued fraction expansion of $1-\gamma$ and $\zeta(n)$.

\section{Introduction and statement results}

We find the expansion Euler's constant in series.

Theorem 1. The following relation holds

$$
\begin{aligned}
1-\gamma= & \frac{1}{4}+\frac{1}{2 \cdot 3}\left|\begin{array}{cc}
1 & \frac{1}{2} \\
\frac{1}{2} & \frac{2}{3}
\end{array}\right|+\frac{1}{3 \cdot 4}\left|\begin{array}{ccc}
1 & \frac{1}{2} & \frac{1}{3} \\
0 & 1 & \frac{1}{2} \\
\frac{1}{2} & \frac{2}{3} & \frac{3}{4}
\end{array}\right|+\frac{1}{4 \cdot 5}\left|\begin{array}{cccc}
1 & \frac{1}{2} & \frac{1}{3} & \frac{1}{4} \\
0 & 1 & \frac{1}{2} & \frac{1}{3} \\
0 & 0 & 1 & \frac{1}{2} \\
\frac{1}{2} & \frac{2}{3} & \frac{3}{4} & \frac{4}{5}
\end{array}\right| \\
& +\frac{1}{5 \cdot 6}\left|\begin{array}{ccccc}
1 & \frac{1}{2} & \frac{1}{3} & \frac{1}{4} & \frac{1}{5} \\
0 & 1 & \frac{1}{2} & \frac{1}{3} & \frac{1}{4} \\
0 & 0 & 1 & \frac{1}{2} & \frac{1}{3} \\
0 & 0 & 0 & 1 & \frac{1}{2} \\
\frac{1}{2} & \frac{2}{3} & \frac{3}{4} & \frac{4}{5} & \frac{5}{6}
\end{array}\right|+\cdots
\end{aligned}
$$

Proof. We transform the integral

$$
\begin{aligned}
& -\gamma=\int_{0}^{1} \ln |\ln x| d x \\
& -\gamma=\int_{0}^{1} \ln |\ln (1-x)| d x
\end{aligned}
$$

and have

$$
1-\gamma=\int_{0}^{1} \ln \left(1+\frac{x^{1}}{2}+\frac{x^{2}}{3}+\frac{x^{3}}{4}+\cdots\right) d x .
$$

We need the lemma.

Received September 4, 2009. 
Lemma 1. The following expansion holds

$$
\begin{aligned}
& \ln \left(1+A_{1} x^{1}+A_{2} x^{2}+A_{3} x^{3}+\cdots\right) \\
& =A_{1} x^{1}+\frac{1}{2}\left|\begin{array}{cc}
1 & A_{1} \\
A_{1} & 2 A_{2}
\end{array}\right| x^{2}+\frac{1}{3}\left|\begin{array}{ccc}
1 & A_{1} & A_{2} \\
0 & 1 & A_{1} \\
A_{1} & 2 A_{2} & 3 A_{3}
\end{array}\right| x^{3} \\
& \quad+\frac{1}{4}\left|\begin{array}{cccc}
1 & A_{1} & A_{2} & A_{3} \\
0 & 1 & A_{1} & A_{2} \\
0 & 0 & 1 & A_{1} \\
A_{1} & 2 A_{2} & 3 A_{3} & 4 A_{4}
\end{array}\right| x^{4}+\cdots
\end{aligned}
$$

Proof of Lemma 1. Let

$$
\ln \left(1+A_{1} x^{1}+A_{2} x^{2}+A_{3} x^{3}+\cdots\right)=b_{0} x^{1}+\frac{1}{2} b_{1} x^{2}+\frac{1}{3} b_{2} x^{3}+\cdots
$$

then

$A_{1}+2 A_{2} x^{1}+3 A_{3} x^{2}+\cdots=\left(1+A_{1} x^{1}+A_{2} x^{2}+A_{3} x^{3}+\cdots\right)\left(b_{0}+b_{1} x^{1}+b_{2} x^{2}+b_{3} x^{3}+\cdots\right)$.

The coefficient $b_{n}$ we find by solving a system of equations

$$
\left\{\begin{array}{cl}
b_{0} & =A_{1} \\
b_{0} A_{1}+b_{1} & =2 A_{2} \\
b_{0} A_{2}+b_{1} A_{1}+b_{2} & =3 A_{3} \\
\vdots & =\vdots \\
b_{0} A_{n}+b_{1} A_{n-1}+b_{2} A_{n-2}+\cdots+b_{n} & =(n+1) A_{n+1} .
\end{array}\right.
$$

Hence

$$
b_{n}=\left|\begin{array}{ccccccc}
1 & A_{1} & A_{2} & A_{3} & \cdots & & A_{n} \\
0 & 1 & A_{1} & A_{2} & \cdots & & A_{n-1} \\
0 & 0 & 1 & A_{1} & \cdots & & A_{n-2} \\
\vdots & \vdots & \vdots & \vdots & \cdots & & \vdots \\
0 & 0 & 0 & 0 & \cdots & 1 & A_{1} \\
A_{1} & 2 A_{2} & 3 A_{3} & 4 A_{4} & \cdots & & (n+1) A_{n+1}
\end{array}\right| .
$$

The proves the Lemma 1.

Application of (3) into (2) gives (1).

We use now the well known formula

$$
=\frac{a_{1}+a_{1} a_{2}+a_{1} a_{2} a_{3}+a_{1} a_{2} a_{3} a_{4}+\cdots}{1-\frac{a_{1}}{1+a_{2}-\frac{a_{2}}{1+a_{3}-\frac{a_{3}}{1+a_{4}-\cdots}}}}
$$


The restriction: The above series is convergent.

Hence the formula (1) can be written in the following form

$$
\begin{aligned}
& 1-\gamma=\frac{\frac{1}{4}}{\frac{1}{2}\left|1 \frac{1}{2}\right|}(5)
\end{aligned}
$$

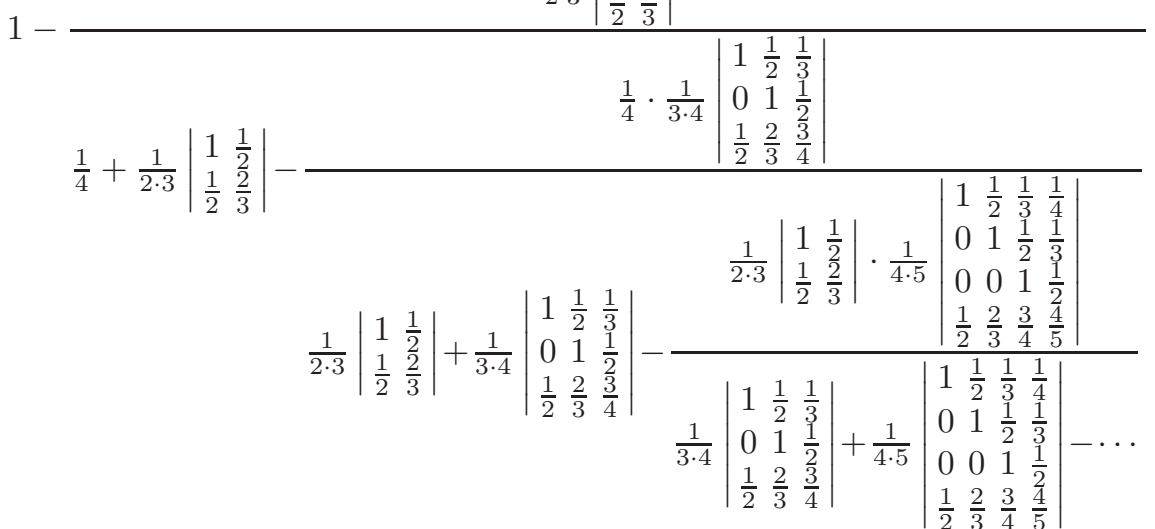

We consider the Riemann zeta function

$$
\zeta(n)=\sum_{k=1}^{\infty} \frac{1}{k^{n}} \quad n=2,3,4, \ldots
$$

Application of (4) leads to the following formula

$$
\zeta(n)=\frac{1}{1-\frac{1^{2 n}}{2^{n}+1^{n}-\frac{2^{2 n}}{3^{n}+2^{n}-\frac{3^{2 n}}{4^{n}+3^{n}-\cdots}}} .}
$$

The formula (7) is the special case of the expansion

$$
\zeta(n ; a)=\frac{1}{a^{n}-\frac{a^{2 n}}{(a+1)^{n}+a^{n}-\frac{(a+1)^{2 n}}{(a+2)^{n}+(a+1)^{n}-\frac{(a+2)^{2 n}}{(a+3)^{n}+(a+2)^{n}-\cdots}}} .}
$$

where

$$
\zeta(n ; a)=\sum_{k=0}^{\infty} \frac{1}{(a+k)^{n}} \quad a>0 .
$$

Proof of (8). It is enough to show that the expression $a^{n}-\frac{1}{\zeta(n ; a)}$ satisfies the recurrential relation

$$
a^{n}-\frac{1}{\zeta(n ; a)}=\frac{a^{2 n}}{(a+1)^{n}+a^{n}-\left[(a+1)^{n}-\frac{1}{\zeta(n ; a+1)}\right]} .
$$


We have

$$
\begin{aligned}
\frac{a^{2 n}}{(a+1)^{n}+a^{n}-\left[(a+1)^{n}-\frac{1}{\zeta(n ; a+1)}\right]} & =\frac{a^{n}\left(a^{n}+\frac{1}{\zeta(n ; a+1)}-\frac{1}{\zeta(n ; a+1)}\right)}{a^{n}+\frac{1}{\zeta(n ; a+1)}} \\
& =a^{n}-\frac{a^{n}}{a^{n} \zeta(n ; a+1)+1} \\
& =a^{n}-\frac{1}{\zeta(n ; a)} .
\end{aligned}
$$

Putting $a=1$ in (8) we obtain (7).

\section{References}

[1] E. T. Whittaker and G. N. Watson, A Course of Modern Analisys, 4th ed., Cambridge University Press, Cambridge, 1927.

ul. Marszałkowska 1 m. 80, 00-624 Warszawa, Poland. 